\title{
EFECTOS NO PREVISTOS EN LAS INNOVACIONES EN EDUCACION: EL PROCESO DE CAMBIO ABIERTO A LA INCERTIDUMBRE ${ }^{132}$
}

\author{
Daniel Ríos Muñoz ${ }^{133}$
}

\section{RESUMEN}

La mayor parte de las acciones desarrolladas en el marco de la Reforma Educacional Chilena buscan promover la acción innovadora de los profesores, con el propósito de que su actividad docente contribuya al mejoramiento de los aprendizajes de los estudiantes. Una de las estrategias más novedosa para despertar la capacidad de innovar de los docentes está constituida por los Proyectos de Mejoramiento Educativo (PME). En esta ponencia se describe y analiza los diferentes resultados no previstos de las innovaciones -independientes de los PME- desarrolladas por profesores de educación básica, y que no estaban contemplados originalmente en sus objetivos. Esta situación hace pensar que el profesor innovador no posee un control pleno en el desarrollo de la innovación, lo que lo obliga a asumir un cierto grado de incertidumbre durante su ejecución. Este hallazgo tiende a cuestionar el modelo de Investigación y Desarrollo que ha orientado diversos procesos innovadores basados en una racionalidad instrumental, y cuyos conceptos claves se relacionan con control, eficiencia y eficacia.

En el caso de los estudiantes, los principales aprendizajes no previstos se relacionan con un mayor desarrollo de características afectivas y actitudinales como: incremento en la participación escolar, mayor identificación con la unidad educativa, aumento en el compañerismo entre alumnos, mejor relación con sus profesores y mejores aprendizajes cognoscitivos. Sin embargo, también se observan efectos no previstos de carácter negativo como: envidia, individualismo, competencia, autoritarismo, indisciplina, entre otros. En los profesores innovadores, se aprecian efectos no previstos vinculados a la aplicación de estrategias de enseñanza más activas, un mayor interés en el perfeccionamiento de temáticas ligadas a la acción de mejoramiento y una mayor comunicación con otros profesores del establecimiento acerca de la innovación ejecutada.

\section{FUNDAMENTOS DEL PROBLEMA}

A partir de la década de los 90 los gobiernos de la Concertación de Partidos por la Democracia han centrado su preocupación en la perspectiva de construir un país que busca equilibrar el crecimiento económico con la ampliación de oportunidades de desarrollo para todos sus ciudadanos, con especial énfasis por aquellos que presentan una mayor carencia social, económica y cultural. Esta opción política, busca desarrollar una nación con una mayor igualdad de oportunidades para las personas, especialmente de niños y jóvenes, de tal forma de favorecer los procesos de integración interna, en el contexto de un sistema democrático que requiere ampliarse y profundizarse con el propósito de consolidarse como forma de gestión gubernamental y de expresión ciudadana.

\footnotetext{
132 Ponencia presentada en el Seminario Nacional La Reforma Educacional en Marcha Análisis y Perspectivas, 24-26 de julio de 2002, Valparaíso, Chile.

133 Docente e investigador, Departamento de Educación, Facultad de Humanidades. Universidad de Santiago de Chile. Correo electrónico:driosm@lauca.usach.cl. 
En el plano educacional, estas orientaciones fundantes, que buscan promover la participación y autonomía de los actores educativos, se han materializado, desde hace más de una década, por parte del Ministerio de Educación a través de diversas intervenciones que han tenido como propósito central el de mejorar la Calidad y Equidad de la Educación. Una de las primeras acciones de gran envergadura se relacionaron con el Programa MECE-BÁSICA, diseñado y puesto en marcha por el MINEDUC en 1991. Esta acción pasó a ser parte de los programas de mejoramiento, como uno de los componentes de la Reforma Educacional Chilena, iniciada por los responsables ministeriales a partir de 1996 con la aplicación de la Reforma Curricular en Educación Básica. Esta gran intervención se ha realizado en todos los niveles del sistema y ha involucrado la combinación de esfuerzos humanos, materiales y financieros de gran magnitud, que lo constituyen como el cambio integral más importante y profundo jamás realizado en el país (Cox, 1997 y García-Huidobro, 1999).

La estrategia más innovadora del MECE-BASICA estuvo constituida por el diseño e implementación de los Proyectos de Mejoramiento Educativo (PME), en el marco de la Descentralización Pedagógica. Los PME, ofrecían una oportunidad de desarrollo de la iniciativa e innovación a los docentes -más allá de su participación en ellos-, destinada a optimizar los procesos de enseñanza y a mejorar, en consecuencia, el nivel de los diversos aprendizajes de los alumnos (conocimientos, habilidades y actitudes). A través de los PME, se reconocía la capacidad autónoma e innovadora del cuerpo docente y la comunidad escolar, para realizar un diagnóstico concreto y directo de la problemática de su unidad educativa, en la perspectiva de mejorar su calidad y la equidad de los servicios que ofrece, en un trabajo eminentemente participativo, cooperativo e integrado, con el fin de ayudar a transformar el quehacer pedagógico de los profesores y promoción de una nueva cultura escolar (Ministerio de Educación, 1994).

Inicialmente el desarrollo de los PME, y de otras acciones del Programa MECEBASICA, fueron una respuesta ante la constatación, en términos de la ineficiencia del sistema, de la alta repitencia de los alumnos de enseñanza básica, especialmente en las zonas de pobreza urbana y rural, ya que los alumnos de escuelas públicas tardan un promedio de 12,4 años, en terminar el ciclo de 8 años de educación primaria, en contraste a los 8,8 años de los niños y niñas de colegios privados pagados (op. cit). De acuerdo al Ministerio de Educación, las principales causas de esta situación académica se relacionan, principalmente, con: la baja pertinencia de los contenidos del currículum con la realidad social, deficiencias metodológicas y baja motivación de los profesores; como también en la desorientación de la supervisión de directores y supervisores en los procesos pedagógicos del aula; carencia de materiales educativos y recursos de las escuelas, (ibid). Desde el punto de vista de las prácticas pedagógicas, Montero, (1988) y Castro, (1993), plantean que éstas se caracterizan por ser: rutinarias y homogenizadoras; apegadas a esquemas rígidos y verticalizados; excluyentes de alternativas; enciclopedistas en sus pretensiones; promotoras del valor de la disciplina, del orden constituido y la obediencia acrítica a la autoridad; desarticuladoras del saber; asignaturista y comprometidas con una visión fragmentaria de la realidad.

En este contexto general, el problema de este estudio se relaciona con las innovaciones que ejecutan los profesores y que buscan responder al tradicionalismo de sus prácticas pedagógicas, las cuales se distinguen por el apego a acciones invariables en ellas, las que han sido construidas, desarrolladas y apropiadas por los profesores a través de los años, pero que no tienden a ser cuestionadas por éstos con vista a una transformación positiva de su labor docente. Uno de los objetivos del estudio se relaciona 
con la identificación y descripción de los efectos no previstos en el diseño y desarrollo de innovaciones aplicadas por los docentes de educación básica.

\section{MARCO CONCEPTUAL}

En los últimos 30 años se puede observar en la literatura respectiva una evolución del término innovación, donde se pude apreciar elementos comunes y otros que marcan una diferenciación de acuerdo a cada autor (Huberman, 1973, Morrish, 1978; Havelock y Huberman, 1980; González y Escudero, 1987; Restrepo, 1995; Sancho, 1998). Los aspectos nucleares de la innovación que vinculan las diversas acepciones de ella, dicen relación con que ésta debe ser: deliberada, planificada, específica y evaluada después de un tiempo suficiente en relación con sus objetivos pedagógicos y sociales, además debe aportar algo distinto o nuevo en el contexto en la cual se aplica.

Subyacente a la mayoría de las primeras conceptualizaciones, es posible apreciar una racionalidad técnica, cuyas principales características se vinculan con aspectos como, eficacia, control, recursos humanos y materiales, metas y objetivos, todos conceptos que son inherentes a la lógica que orienta los procesos involucrados en la industrialización de los países desarrollados, de hace algunas décadas atrás, en el contexto de una fuerte interdependencia entre ciencia y tecnología. Así, esta perspectiva se concreta en modelos ad-hoc, por las cuales se guían las primeras innovaciones en el ámbito educativo, condicionadas por las etapas que utilizan las innovaciones industriales: Investigación; Desarrollo y Difusión. Todo el proceso innovador tiene como propósito final el cumplimiento de los objetivos preestablecidos y su logro es considerado como el éxito buscado a través de las acciones planificadas con precisión.

A este tipo de innovaciones, Tack (citado en Moreno, 1996) las denomina instrumentales, y sólo proporcionan medios de realización más efectivos y menos oneroso para el logro de ideas que se concretizan en determinados objetivos. Sin embargo, a este sustrato instrumental de las primeras definiciones, y sus correspondientes aplicaciones, aparece un elemento que permite ampliar y profundizar el tema de las innovaciones en el ámbito educativo. La idea nueva se refiere a lo que plantea Morrish (1978), entre otros, en cuanto a que éstas deben valorarse no sólo en relación con los objetivos pedagógicos establecidos en el proyecto, sino también a sus objetivos sociales, produciéndose una relación lógica y de dependencia entre ambos, lo que permite otorgarle a las innovaciones una trascendencia que supera la dimensión escolar y que tiene un impacto multiplicador en la comunidad en la cual se inserta el centro educativo.

En definitiva, a partir de las acepciones de la innovación desarrolladas por Morrish (1978), González y Escudero (1987), Restrepo (1995), Imbernón (1995), Moreno, (1996), entre otros, comienza a desarrollarse una concepción más política y socio-cultural de las innovaciones, que se basa en principios, entre otros, como: autonomía, participación, cooperación entre los actores educativos, toma de decisión en conjunto, y una clara conciencia en éstos del entorno social y natural en el cual se desarrollan aquellas. Así, desde las diversas aproximaciones conceptuales del término innovación -que epistemológicamente se orientan por paradigmas de racionalidad diferentes- se proponen consecuentes modelos para su elaboración, desarrollo y evaluación, como se detalla a continuación.

\section{Modelos de innovaciones}

En general, la literatura reconoce cuatro grandes orientaciones para la elaboración, 
ejecución y evaluación de las innovaciones en el ámbito escolar: modelo de investigación, desarrollo y difusión; modelo de interacción social; modelo organizativo; modelo de resolución de problemas.

\section{Modelo de investigación y desarrollo.}

Este modelo orienta las primeras innovaciones educativas puestas en marcha y está fuertemente condicionado por las innovaciones ejecutadas en el campo de las ciencias, tecnología e industria de los países desarrollados. Se caracteriza por tener un enfoque lógico y racional que busca resolver un determinado problema, detectado por profesionales externos -individuales e institucionales- a la escuela, a través de una estrategia que se considera como la más adecuada. Tiende a ser fuertemente asimétrica, dado que el grado de colaboración entre los que realizan el diseño de la innovación y los ejecutantes -profesores- es baja o nula, lo que determina una clara distinción de roles y funciones.

Así, los especialistas (diseñadores) deciden que es lo conveniente de poner en marcha y los docentes son considerados técnicos que ejecutan las diversas acciones contempladas en la mejora elaborada por aquellos. Estas acciones plantean una secuencia lineal y cada actividad presenta un fino detalle para su ejecución, por lo que la función del profesor se limita al cumplimiento racional de lo previamente establecido por otros, independiente de su interés y necesidad de cambiar. Las innovaciones exitosas desarrolladas a la luz de este modelo se validan externamente a través de su difusión y adopción por otros interesados. Estas fases incorporan actividades de diseminación, demostración, prueba, implantación e institucionalización definitiva (Morrish, 1978).

\section{Modelo de interacción social}

Se basa en la observación y juicio de los posibles usuarios de la innovación que es formulada y ejecutada en una realidad escolar diferente a la de éstos. Los posibles adaptadores, a través de la relación personal y profesional que establecen con otros innovadores, toman conocimiento de la ejecución de una determinada innovación, estimando la posibilidad de replicarla en su propio ámbito educativo, al poseer una problemática similar al contexto donde se aplica ésta. Este modelo es utilizado en las escuelas demostrativas y entre sus fases se encuentra la toma de conciencia, el interés, la evaluación, el ensayo y la adopción.

\section{Modelo Organizativo}

El modelo organizativo se plantea desde un enfoque cultural, considerando a la escuela como una organización que posee una cultura propia y distintiva. Está basado en la dinámica de las organizaciones, cuya característica principal se relaciona con la constitución de entramados de personas y grupos que se vinculan de diversas formas al interior del centro educativo, lo que puede favorecer o no el cumplimiento de los fines y objetivos institucionales. (Zaltman, et al., 1977 y Brow y Moberg, 1980, en Ferrández y Puente, 1992). Las principales fases de este modelo son: la planificación, la adopción, la ejecución y la evaluación. 


\section{Modelo Solución de Problemas}

Este modelo también se presenta desde una perspectiva cultural y tiene como punto de partida y como eje central a los profesores, en tanto que son los que definen las necesidades iniciales y actúan solos o con asesores. Estos pretenden reestructurar internamente las condiciones desfavorables que presenta la labor educativa de la escuela y su participación es esencial para el logro de la innovación formulada. El agente externo puede asumir el rol de colaborador de la experiencia de mejoramiento, pero no tiene el protagonismo que se espera de los profesores. La interacción y reconocimiento mutuo de éstos, es la condición necesaria para el éxito de la innovación aplicada (Morrish, 1978, Huberman, 1984). En su proceso se distinguen las siguientes etapas: la valorización del docente, el diagnóstico, el rol del asesor externo, el conocimiento del profesor y la motivación del profesor.

En resumen, los principales modelos que han guiado las innovaciones pedagógicas en los últimos años, tanto de aquellas que son elaboradas bajo una orientación técnica, como las diseñadas desde una perspectiva cultural, contemplan en su diseño, diversas etapas que guardan relación con:

a) el reconocimiento, por los actores de la escuela, de algún problema o crisis al interior de ella.

b) la elaboración y ejecución de estrategias innovadoras para su solución.

c) la retroalimentación constante de las acciones innovadoras.

d) la valoración de los profesores involucrados en los procesos de renovación, tanto en lo que se refiere a los conocimientos construidos durante su trayectoria profesional como en sus motivaciones intrínsecas para participar activamente en acciones de transformación de su práctica pedagógica.

Por otro lado, en los modelos culturales y solución de problemas es posible reconocer, al menos, dos aspectos que superan la visión técnica de las primeras innovaciones puestas en marcha en el ámbito educativo formal, bajo la orientación del modelo Investigación y Desarrollo. En primer lugar, se reconoce a los profesores como los principales agentes del cambio, lo que permite inferir que el éxito o fracaso de las innovaciones tienen en ellos un rol fundamental, es decir, sin su participación y compromiso las innovaciones escolares probablemente tienden a no alcanzar sus propósitos. En esta línea, se pone de relieve el saber pedagógico de cada profesor y profesora; sus motivaciones más íntimas para comprometerse con mejoras, que, directa 0 indirectamente, remecen sus tradiciones emergidas a través del tiempo: éstas constituyen sus rutinas escolares, con ellas logra una cierta tranquilidad en su actuación docente que, sólo se ve amenazada cuando son confrontadas con la posibilidad de cambio, mediante la ejecución de innovaciones.

Y, en segundo lugar, las innovaciones no sólo pueden estar referidas al mejoramiento de los aprendizajes de los alumnos ocurridos al interior de la sala de clases, sino también, a cualquier aspecto o variable que el conjunto de los actores de la comunidad considere adecuada abordar, a través de estrategias pertinentes y consensuales. En consecuencia, las innovaciones pueden trascender el ámbito del aula, pudiendo ser ejecutadas en cualquier dimensión de la escuela o en aquellas áreas en que ésta se vincula con la comunidad a la cual pertenece. Sin embargo, y dadas las funciones que la sociedad le ha confiado, toda innovación debe apuntar, directa o indirectamente, a elevar y profundizar la calidad de los aprendizajes de los estudiantes. 


\section{METODOLOGIA}

La investigación se realiza en escuelas primarias de Santiago, posee características del tipo exploratorio-descriptivo y se utilizó un diseño que contempla la presencia de un grupo de profesores que participaron en los Proyectos de Mejoramiento Educativo (PME), en educación básica, y un grupo de docentes que no lo hicieron, el que sirve de comparación, siguiendo el diseño de comparaciones de mediciones de postest de ambos grupos de Bingham y Felbinger (1989). A ambos grupos se les aplica un cuestionario semiestructurado y los contenidos de las respuestas se analizan, en primer lugar, por medio de estadística descriptiva y, posteriormente, se realiza un análisis interpretativo de ellos. Enseguida se efectúa una discusión de los resultados a la luz del marco conceptual adoptado, con un especial énfasis en los aspectos que se postulan en este estudio acerca de la innovación en educación.

\section{RESULTADOS}

\section{Efectos esperados}

Como se señala en Ríos (2001), los principales resultados de las innovaciones ${ }^{134}$ desarrolladas por los profesores de educación básica ${ }^{135}$ se vinculan con sus objetivos declarados: mejores aprendizajes en las áreas socioafectiva y cognoscitiva de los alumnos. Se aprecia una mayor autonomía, motivación, capacidad de decisión, valoración del medio ambiente y autoestima, entre otros, y un mejoramiento de la comunicación oral y escrita y del cálculo en operaciones básicas en matemática, además de mejores aprendizajes en disciplinas como ciencias sociales e idioma inglés. También expresan que es posible visualizar un mayor desarrollo en aspectos como: el respeto entre ellos y una mejor comunicación con sus profesores; apoyo de los padres en los aprendizajes de sus hijos y la detección de estudiantes con aptitudes artísticas y deportivas. Estos resultados son similares a los encontrados en otros estudios relacionados con las innovaciones educativas (Fuentelabrada y Nemrovsky, 1988; Nogales, 1988; Ghani, 1992; Colbert, et al., 1992; Ramírez y Sánchez, 1995). Por otra parte, es interesante destacar que algunos docentes innovadores señalan que no se observan resultados o se manifiestan en forma escasa y otros expresan que aún, al momento del estudio, no evalúan a pesar que esas acciones se desarrollan desde hace un tiempo.

Como la mayor parte de estos resultados se relacionan directamente con los objetivos explícitos de las innovaciones, hace pensar que los docentes han tenido un determinado control sobre las acciones innovadoras realizadas, las que a su vez han sido bien escogidas para alcanzar tales propósitos, al igual que los materiales y recursos utilizados. En este contexto, es posible considerar que el diseño y la ejecución de una innovación, al ser una actividad que está orientada por una determinada racionalidad, tenderá a ser exitosa en la medida que la estrategia escogida sea la pertinente para superar el problema o dificultad que aborda, que cuente con materiales adecuados y que el innovador que la ejecuta posea la capacidad de regularla de manera permanente. Por

\footnotetext{
${ }^{134}$ Cabe recalcar que las innovaciones detectadas en este estudio son independientes y distintas de la ejecución de los PME, en el caso de los profesores de este grupo.

135 Dado que en el análisis de las características de las innovaciones no se observaron mayores diferencias entre los profesores PME y los del grupo de comparación, se postula que los innovadores conforman un solo grupo, hayan o participado en los Proyectos de Mejoramiento Educativo, lo que conlleva a presentar los resultados de manera agrupada. 
otro lado, un factor que también estaría ligado al éxito de las innovaciones sería el tiempo, cuya cantidad necesaria, probablemente, se relacione con la naturaleza de la innovación, la comprensión que tengan los participantes de ella y el control que se ejerza en su orientación, entre otros aspectos (Ríos,2000). Este planteamiento podría explicar el hecho de que algunos docentes expresen que no observan logros en sus innovaciones o que se aprecian escasamente.

\section{Efectos no esperados}

A estos resultados esperados, se presentan en las innovaciones ejecutadas efectos no previstos, tanto positivos como negativos, los cuales pueden ser considerados como uno de los hallazgos más importantes de este estudio. Entre los principales resultados no esperados de carácter positivo, se pueden mencionar los siguientes: mayor desarrollo de características afectivas y actitudinales de los alumnos como: motivación, participación, seguridad personal, autoestima, asistencia a clases e identificación con la escuela $(37 \%)^{136}$; mayor fraternidad y sociabilidad entre los alumnos y de éstos con sus profesores y padres (27\%); mejores aprendizajes cognoscitivos de los estudiantes como: incremento en las calificaciones, mayor comprensión sobre lo que están aprendiendo y aumento en la capacidad crítica $(11 \%)$. Por parte de los profesores: realización de clases más activas, interés por nuevos perfeccionamientos y aumento en el intercambio de experiencias con otros docentes (10\%); mayor preocupación y apoyo de los padres y apoderados en los aprendizajes de sus hijos (09\%); no ha observado efectos no previstos o no sabe $(06 \%)$.

Gráficamente estos efectos no previstos de las innovaciones pueden observarse en la siguiente figura:

\section{Efectos positivos no previstos de las innovaciones}

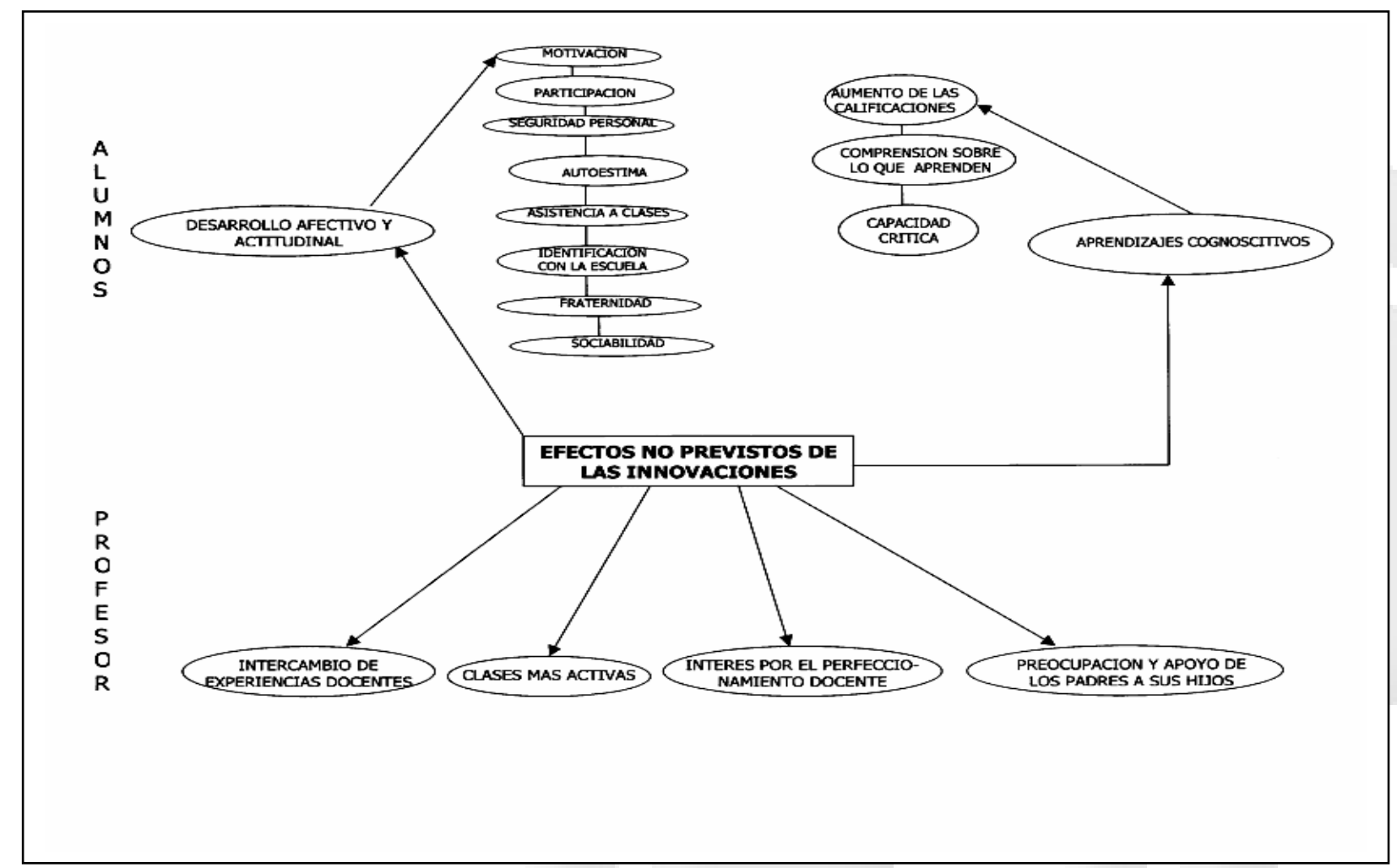

136 Corresponde a las respuestas comunes de los entrevistados, a cada pregunta del cuestionario aplicado, las que se ordenan de mayor a menor según los porcentajes de éstas. Estos porcentajes se obtienen a partir de la totalidad de las menciones realizadas por los entrevistados, los que pueden realizar más de una respuesta, provocando con ello, que el número total sea superior al número de profesores innovadores de este estudio (55). En este caso las menciones fueron 79. 
El hecho que las innovaciones presenten efectos que no estaban inicialmente considerados hace pensar que, a pesar de que éstas están orientadas por objetivos previamente establecidos, existe un determinado grado de incertidumbre en su aplicación que impide un control pleno por parte del profesor. Esta situación de inseguridad puede condicionar la eficacia de las innovaciones, ya que es posible que en el caso de algunos procesos innovadores los resultados no previstos sean de mayor importancia, en términos educativos, que el cumplimiento de los objetivos previamente declarados.

Por otro lado, Algunos efectos no previstos, a diferencia de los esperados en los objetivos, son negativos, principalmente en el caso de los estudiantes:

"Actitudes negativas por parte de algunos estudiantes como envidias, individualismo y competencia." (sujeto 1)

"...Actitudes negativas generadas en algunos alumnos como autoritarismo, egoísmo y envidias." (sujeto 11)

"Mayor indisciplina" (sujeto 41)

Este tipo de efecto en los alumnos puede ser un indicador de la falta de comprensión por parte de éstos acerca de la naturaleza y propósitos de la innovación, de un escaso interés o baja motivación, situación que podría ser disminuida por la explicación y reflexión permanente del profesor con los beneficiados de ella, a fin de otorgarle sentido a la acción de cambio positivo. Por otra parte, estos resultados debieran servir de base para que el docente oriente la innovación desde una racionalidad más comprensiva que instrumental, guiándose más bien por los modelos culturales y solución de problemas, asumiendo que el aspecto subjetivo de los involucrados en ella -valoración de la innovación, actitudes, motivación, sentimientos, etc.- puede jugar un papel más importante que los factores materiales, didácticos, racionales y explícitos presentes en la transformación, enfoque que debiera, en consecuencia, favorecer el diálogo, la reflexión y la negociación de significados entre los participantes de la innovación.

Esta situación es posible de observar con relación a algunos de los efectos no previstos que se presentan en los propios profesores que innovan, a pesar que ninguna de las innovaciones analizadas tiene explícitamente considerada en los objetivos beneficios para ellos:

"...El trabajo del profesor se hace más liviano por el hecho de usar material didáctico como las fichas." (sujeto 7)

"Trabajo del docente más aliviado con el uso de material didáctico elaborado y con la participación de alumnos-monitores" (sujeto 11)

"Autorrealización del docente." (sujeto 25)

Este impacto en los innovadores se puede apreciar en dos niveles: pragmático y personal. El primero, se refiere a un aspecto de su práctica pedagógica como lo es la metodología -y la importancia de contar con material didáctico-, efecto que incluso puede ayudarle a modificar conceptualmente el rol que le compete al profesor en el proceso de enseñanza. $Y$, el segundo, se relaciona con la satisfacción más intima que proporciona el desarrollo y resultados de la innovación, lo cual le otorga, probablemente, un mayor sentido a su profesión, una apertura a otros docentes y una búsqueda permanente por el mejoramiento de su quehacer pedagógico, tal como algunos de ellos lo expresan:

"Interés de otros profesores por la innovación aplicada..." (sujeto 7)

"Interés de otros profesores en apoyar a los alumnos-monitores..." (sujeto 36)

"Valoración de la renovación metodológica del profesor..." (sujeto 25)

El otro efecto no previsto, y quizás el más importante por su trascendencia formativa, se vincula con el mejoramiento de las relaciones entre los propios estudiantes y de éstos con sus profesores y padres, tal como se puede apreciar en sus propios comentarios: 
"Alumnos más sociables y comunicativos entre ellos y con sus profesores, en especial, aquellos que han logrado éxito en su proceso de aprendizaje." (sujeto 9) "Mejoramiento de la convivencia entre alumnos y de éstos con el profesor. Menor frustración en algunos estudiantes" (sujeto 17)

"Mejor relación entre alumnos y entre éstos y los padres y apoderados. Mejor rendimiento escolar y un mayor interés de los alumnos en sus estudios." (sujeto 37)

El mejoramiento de la calidad en las relaciones interpersonales de los diferentes actores escolares involucrados en la innovación, puede constituir un aspecto fundamental para explicar los diferentes efectos positivos no esperados, y probablemente también de aquellos que estaban previstos en los objetivos del cambio, situación que podría estar relacionada con el clima cálido en el cual se desarrolla la innovación -aspecto que podría estar vinculado a características personales de los profesores-, participación, compromiso e interés de alumnos y padres y la comprensión por parte de éstos acerca de su finalidad y utilidad.

En este sentido, parece ser esencial la percepción positiva que tienen los alumnos del aporte que pueden realizar sus padres y apoderados, para lograr mejores aprendizajes:

"Los alumnos perciben que son apoyados por sus padres en su formación. Padres que trabajan mejor y de manera más fácil con sus hijos." (sujeto 12)

"Alumnos más satisfechos de su desarrollo personal [por la] Incorporación de los padres al proceso educativo." (sujeto 32)

\section{A MODO DE CONCLUSIONES}

Los resultados esperados de acuerdo a los objetivos de cada innovación son acompañados por efectos no previstos. Estos resultados no esperados por el innovador, se presentan mayoritariamente de manera positiva, principalmente en los estudiantes. En éstos se reconoce un incremento en la asistencia a clases, un mayor compromiso y responsabilidad en los estudios, una mayor autorregulación en el comportamiento escolar, un mejoramiento en la autoimagen que otorga un aumento en la seguridad personal, un incremento en la fraternidad entre pares y una mayor capacidad crítica, entre otros aprendizajes. En el caso de los profesores, éstos reconocen que la elaboración y aplicación de nuevos materiales didácticos les ha facilitado el trabajo con los estudiantes y el intercambio de experiencias con otros docentes, pero también se presentaron, entre otros costos, repercusiones económicas, ya que ellos mismos debieron solventar su financiamiento.

Estos efectos no previstos en los objetivos de las innovaciones, muestran que ellas no son posibles de controlar plenamente por parte del innovador, lo que tiende a cuestionar la racionalidad que subyace en aquellas que se orientan bajo el modelo tecnológico y sus conceptos derivados de control, eficiencia y eficacia. Esta apertura de las innovaciones a la incertidumbre podría ser explicada, en parte, por los factores complejos que se presentan en la naturaleza personal de los actores educativos comprometidos en el cambio positivo, y que se manifestarían como condicionantes que obstaculizan la ejecución de las transformaciones desarrolladas. Entre estas características personales se pueden señalar las actitudes de apatía, desinterés, envidias, falta de compromiso, entre otras, en los profesores, directivos, alumnos y padres y apoderados que participan en ellas (Ríos, 2000). 
Estas actitudes negativas, pueden potenciarse, sí la comprensión de la naturaleza y finalidad de la innovación es baja por parte de los que participan en ella, situación que puede conducir a un activismo incesante sin un referente conceptual que oriente las acciones de mejoramiento. En este sentido, las innovaciones pueden adquirir una naturaleza instrumental, ya que se presenta poca o ninguna reflexión profunda de la acción que se ejecuta. Sus participantes hacen cosas pero sin preocuparse por saber el cómo ni para qué esas cosas se hacen, pues subyace, probablemente de manera inconsciente, en ellos una racionalidad tecnocrática.

Para disminuir el grado de incertidumbre que se presentaría en el desarrollo de la innovación, sería necesario incorporar acciones de seguimiento durante el transcurso de ella, lo que permitiría el diálogo y la reflexión entre sus responsables y usuarios, dando lugar a un intercambio de representaciones y sentidos que podrían asegurar una comprensión permanente de los objetivos y naturaleza del cambio positivo y un mayor control sobre los factores que favorecen su ejecución $y$, principalmente, de los que tienden a obstaculizarla. Esta fase de seguimiento no está contemplada en ninguno de los modelos que la literatura señala como referentes para el diseño y ejecución de innovaciones en educación.

\section{BIBLIOGRAFIA}

1. BINGHAM, R. y FELBINGER, C. Evaluative in practice. New York: Longman, 1989.

2. CASTRO, E.. Riesgos y promesas del curriculum de colaboración en contextos de descentralización educativa. Revista Iberoamericana de Educación, volumen 3, 1993, pp. 63-87.

3. COLBERT, et.al The new school program: more and better primary education for children in rural areas in Colombia. Washington: Banco Mundial, 1992.

4. COX, C. La reforma de la educación chilena: contexto, contenidos e implementación. Santiago: Preal, 1997.

5. ESCUDERO, J. La innovación y la organización escolar. En R. Pascual (Coord.). La gestión educativa ante la innovación y el cambio. Madrid: Narcea, 1988.

6. ESCUDERO, J. Formación de centros e innovación educativa. Cuaderno de Pedagogía, $N^{\circ} 220,1993$, pp. 81-84,

7. FERRANDEZ, A. y PUENTE, J. Educación de personas adultas. Psicopedagogía y microdidáctica, Zaragoza: Diagrama, 1992.

8. FUENTELABRADA, I. y NEMIROVSKY, M. Formación de maestros e innovación didáctica. México D.F.: Inp, 1988.

9. GARCÍA-HUIDOBRO, J. (Ed.) La Reforma Educacional Chilena. Madrid: Popular, 1999.

10. GHANI, Z. The use of participatory, school-based, and comunity supported innovations in formal basic education. Ottawa: Idrc, 1992.

11. GONZALEZ, M. y ESCUDERO, J. Innovación educativa. teorías y procesos de desarrollo, Barcelona: Humanitas, 1987.

12. HAVELOCK y HUBERMAN, Innovación y problemas de la educación. Ginébra: UNESCO, 1980.

13. HUBERMAN, A.M. Cómo se realizan los cambios en la educación: una contribución al estudio de la innovación. Paris: UNESCO, 1973.

14. IMBERNÓN,F. Formar para innovar. Cuaderno de Pedagogía, N $N^{\circ} 191,1991$, pp. 71-74. 
15. MINISTERIO DE EDUCACIÓN Orientaciones básicas, objetivos y componentes del programa MECE. Santiago: Mineduc, 1994.

16. MONTERO, P. Empleo y educación: algunos requerimientos para la innovación educacional. Santiago: Orealc, 1988.

17. MORENO, M. Innovaciones pedagógicas. Una propuesta de evaluación crítica. Santafé de Bogotá: Magisterio, 1994.

18. MORRISH, J. Cambio e innovación en la enseñanza. Salamanca: Anaya, 1978.

19. NOGALES, I. Los maestros y las perspectivas de innovación pedagógica. La Paz: Ciid, 1988.

20. RÁMIREZ, P y SÁNCHEZ, G. Escuela Activa. Bogotá: Exe, 1995.

21. RESTREPO, B. La colaboración entre investigadores e innovadores, clave para potenciar el desarrollo y la productividad de la innovación. Santafé de Bogotá: Secab/Colciencias/Menfes, 1995.

22. RíOS, D. Profesores innovadores e innovaciones desarrolladas en escuelas básicas de Santiago. Boletín de Investigación Educacional, Volumen 15, 2000, pp. 408-418.

23. RÍOS, D. Innovative teachers: a permanent learning process to improve teaching and student's learning. En Yearbook on Teacher Education, CD-ROM, 2001.

24. SANCHO, J. y Otros Aprendiendo de las innovaciones en los centros. Barcelona: Octaedro, 1998.

25. SANSANO, et al. El trabajo docente y la renovación de la escuela. Cuaderno de Pedagogía, 220, 1993, pp. 40-43. 